\title{
«Die Kommunikation verändert sich laufend»
}

\author{
Interview: Bruno Kesseli
}

Dr. med. et lic. phil, Chefredaktor

Seit 2007 war Jacqueline Wettstein als Kommunikationsverantwortliche an vorderster Front für die FMH engagiert. Nun hat sie eine neue berufliche Weichenstellung vorgenommen. Im folgenden Interview zieht sie eine kurze Bilanz ihrer Tätigkeit im Dienst der Ärzteschaft, die auch den intensiven, nahezu täglichen Kontakt mit der SÄZ-Redaktion umfasste. Aus diesem Grund wird im Gespräch für einmal auf das formale «Sie» verzichtet.

\begin{abstract}
Während zehn Jahren hast du als Abteilungsleiterin die Kommunikation der FMH mitgeprägt. Mit welchen Vorstellungen hast du die Stelle im Jahr 2007 angetreten?

Das Gesundheitswesen war mir von früheren beruflichen Tätigkeiten bei einem Krankenversicherer und in einer Public-Relations-Agentur bereits bekannt, ebenso die Schweizerische Ärztezeitung. Neu waren für mich sowohl das Verbandswesen und die Funktions-
\end{abstract}

"Aus meiner Sicht hat es somit durchaus Vorteile, wenn die Kommunikationsverantwortlichen zwar auf das medizinische Fachwissen zurückgreifen können, jedoch nicht selbst Ärztinnen oder Ärzte sind.»

weise einer nationalen Berufsorganisation als auch der politische Kontext. Ich hatte die Gelegenheit, bereits vor Stellenantritt etwas FMH-Luft zu schnuppern im Rahmen eines Nachdiplomstudiums bzw. einer Abschlussarbeit. Das hat mir den Einstieg sicherlich erleichtert. Ich habe mich auf spannende Aufgaben in einem interessanten Umfeld gefreut. Und rückblickend kann ich sagen, dass die FMH ihr Versprechen vollumfänglich eingelöst hat.

War es schwierig, als Nicht-Medizinerin in die Komplexität des Gesundheitswesens einzutauchen? Um als Berufsverband in der Politik, in den Medien, aber auch in der Öffentlichkeitsarbeit erfolgreich zu bestehen, sind neben medizinischem Wissen auch juristisches, ökonomisches, statistisches Know-how sowie weitere Fachkenntnisse etwa in eHealth oder Pu-

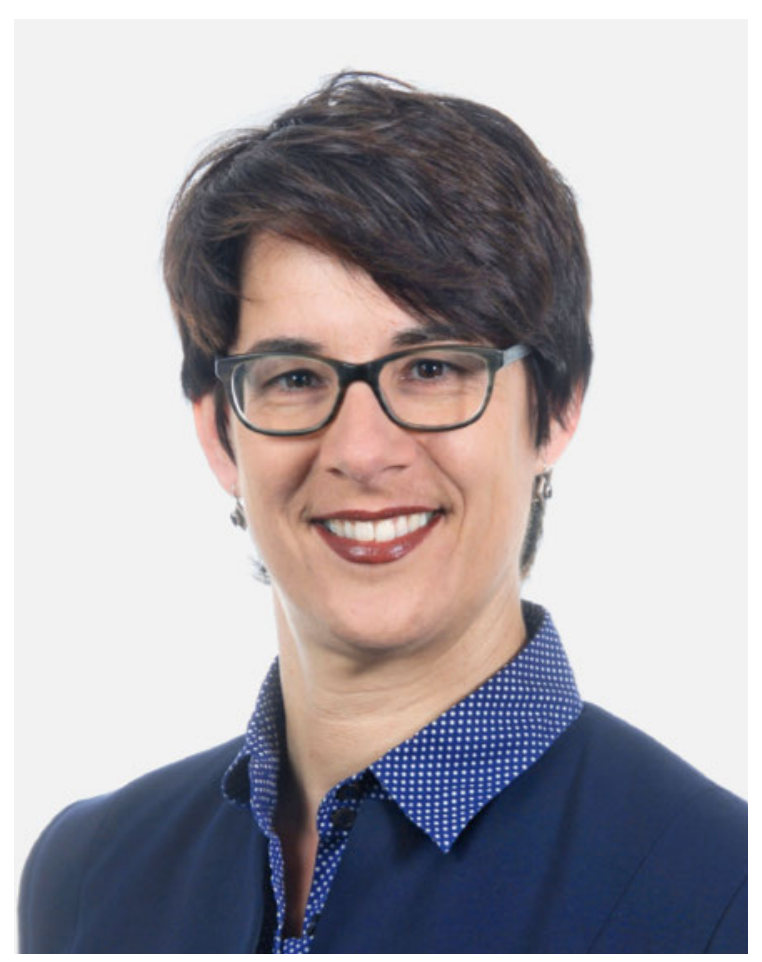

Abschied nach 10 Jahren im Dienst der FMH: Jacqueline Wettstein.

blic Health gefragt. Auf dieses Wissen konnten wir als kommunikationsverantwortliches Team dank den verschiedenen Abteilungen und Diensten des FMHGeneralsekretariates sowie den Basis- und Fachgesellschaften stets zurückgreifen. Es gehört mit zu den zentralen Aufgaben einer Kommunikationsabteilung, das interne Expertenwissen in eine verständliche Sprache zu übersetzen, damit es auch nachvollziehbar wird. 
Aus meiner Sicht hat es somit durchaus Vorteile, wenn die Kommunikationsverantwortlichen zwar auf das medizinische Fachwissen zurückgreifen können, jedoch nicht selbst Ärztinnen oder Ärzte sind.

\section{Wie hat sich die Kommunikation der FMH in den vergangenen zehn Jahren entwickelt?}

Grob vereinfacht würde ich von zwei Phasen sprechen: In meinen ersten FMH-Jahren lag der Schwerpunkt vor allem darin, die Medienarbeit weiter auszubauen sowie die Kommunikation zum Beispiel gegenüber den ange-

"Aus meiner Sicht ist es ein absolutes Plus, dass das 'gelbe Heftli' nicht nur das offizielle FMH-Verbandsorgan ist, sondern gleichzeitig die Pluralität der Ärzteschaft widerspiegelt und somit eine Diskussionsplattform bildet.»

schlossenen Ärzteorganisationen zu verstärken. In meinen späteren Jahren beim Berufsverband haben wir vor allem die Aktivitäten in Public Affairs weiter professionalisiert und optimiert, um so den Gesetzgebungsprozess möglich frühzeitig mitgestalten zu können.

\section{Wie oft musstest du erklären, was die drei Buchstaben FMH bedeuten?}

Kaum je. Auch wenn der volle lateinische Wortlaut nicht geläufig ist, so assoziiert die Öffentlichkeit die drei Buchstaben rasch mit Arzt, Medizin bzw. Gesundheitsversorgung und wertet sie überwiegend positiv. Dies bestätigte auch eine Umfrage bei der Bevölkerung, welche die FMH im Jahr 2014 durchführte. Die Abkürzung «FMH» steht somit für eine starke Marke bzw. ein überzeugendes Label. Sowohl die Bekanntheit als auch das Renommee der FMH tragen wesentlich dazu bei, dass die Meinung des ärztlichen Berufsverbandes gefragt ist, zum Beispiel von Politikern, Medienschaffenden, aber auch von der Öffentlichkeit.

\section{Eine Frage, die mich aus der SÄZ-Perspektive interes- siert: Hat es dein Berufsleben komplizierter gemacht, dass die SÄZ sowohl offizielles Organ der FMH mit einem «Amtsteil» als auch eine Zeitschrift mit einer unabhängigen Redaktion ist?}

Die Schweizerische Ärztezeitung ist eine etablierte Publikation mit Tradition, welche neben Ärztinnen und Ärzten auch Politiker, Medienschaffende sowie weitere Gesundheitsakteure zu ihrer Leserschaft zählt Immer wieder orientieren sich Medienschaffende beispielsweise auch an Themen und Beiträgen der Schweizerischen Ärztezeitung. Als Kommunikationsverantwortliche habe ich den FMH-Teil der Schweizerischen
Ärztezeitung sehr geschätzt, weil dieser dem Berufsverband die Möglichkeit gibt, seine Anliegen regelmässig in einem breit abgestützten Medium vorzustellen. Aus meiner Sicht ist es ein absolutes Plus, dass das "gelbe Heftli» nicht nur das offizielle FMH-Verbandsorgan ist, sondern gleichzeitig die Pluralität der Ärzteschaft widerspiegelt und somit eine Diskussionsplattform bildet.

Welche Ereignisse oder Aspekte deiner Arbeit werden dir als Highlights in Erinnerung bleiben?

Da gab es viele: zum Beispiel meine erste Abstim mungskampagne im Jahr 2008 oder auch die Workshops mit den Kommunikationsverantwortlichen der verschiedenen Ärzteorganisationen. Das letzte grosse Highlight war sicherlich die FMH-Medienkonferenz zum zweiten bundesrätlichen Tarifeingriff, welche von zahlreichen Medienschaffenden besucht wurde und zu entsprechenden Beiträgen führte.

\section{Wo liegen in deiner Erfahrung die grossen Stärken $\operatorname{der}$ FMH?}

Mit über 40000 Mitgliedern ist die FMH hierzulande einer der grossen nationalen Berufsverbände. Die breite Abstützung innerhalb der Ärzteschaft trägt mit dazu bei, dass die FMH zu den zentralen Gesundheitsakteuren zählt. Davon zeugen beispielsweise die Medienanfragen oder auch die Einladungen zu Anhörungen. Die

Die Kunst besteht für den obersten ärztlichen Berufsverband u.a. darin, die Heterogenität der Mitglieder und die unterschiedlichen Meinungen der verschiedenen Ärzteorganisationen zu bündeln.

Kunst besteht für den obersten ärztlichen Berufsverband u.a. darin, die Heterogenität der Mitglieder und die unterschiedlichen Meinungen der verschiedenen Ärzteorganisationen zu bündeln. In diesem Sinne wünsche ich der FMH, dass sie künftig gegen aussen noch vermehrt mit einer einzigen kräftigen Stimme wirksam auftreten kann.

Worauf sollte die Kommunikation der FMH in deinen Augen besonders fokussieren in den nächsten Jahren? Zum einen gilt es, die bestehenden Aktivitäten weiterhin zu überprüfen und zu optimieren. Zum anderen empfiehlt es sich, die Mitgliederkommunikation weiter auszubauen und sie noch stärker auf die Bedürfnisse von Ärztinnen und Ärzten auszurichten. Die Kommunikation verändert sich aufgrund von technischen Entwicklungen laufend: Als Konsumenten wollen wir beispielsweise möglichst zeitgleich zu einem 
Ereignis informiert sein sowie partizipieren können. Ebenso möchten wir News nicht mehr nur in Text aufbereitet bekommen, sondern sie auch mit Bild und Ton erleben können. Die Kommunikationsmöglichkeiten sind heute vielfältiger denn je: Die FMH steht somit vor der spannenden Wahl, ihre Inhalte und Kommunikationskanäle noch besser auf die unterschiedlichen Anspruchsgruppen auszurichten und den Dialog mit diesen zu pflegen.

\section{Wo zieht es dich nach der FMH hin?}

Das Gesundheitswesen ist äusserst facettenreich. Deshalb war für mich klar, dass ich weiterhin in diesem Bereich arbeiten wollte. Seit September leite ich die Kommunikationsabteilung der Integrierten Psychiatrie Winterthur - Zürcher Unterland (ipw). Ich freue mich

«Fehlen wird mir sicherlich die kulturelle und sprachliche Vielfalt, die ich in einer nationalen Organisation während meiner Berner Jahre erleben durfte.»

sehr, das Fachgebiet Psychiatrie näher kennenzulernen und die Funktionsweise einer Institution zu erleben, welche mit Erfolg seit mehr als einem Jahrzehnt einen integrierten Behandlungsansatz verfolgt: So verfügt die ipw an 15 verschiedenen Standorten sowohl über ambulante, tagesklinische als auch stationäre Angebote. Und ebenso deckt sie alle psychiatrischen Krankheitsbilder vom jungen Erwachsenen bis ins hohe Alter ab. Spannend finde ich auch, dass die ipw als Arbeitgeberin sich klar für Teilzeitarbeit auf allen Hierarchiestufen sowie für die Vereinbarkeit von Beruf und Familie engagiert. Als erste psychiatrische Klinik der Schweiz erhielt sie deshalb im Jahr 2016 das Label "Familie und Beruf». Ich freue mich nach der FMH somit auf drei neue Buchstaben, spannende Themen und Aufgaben am Puls des Gesundheitswesens.

\section{Was wirst du vermissen? Was wirst du nicht vermissen?}

Fehlen wird mir sicherlich die kulturelle und sprachliche Vielfalt, die ich in einer nationalen Organisation während meiner Berner Jahre erleben durfte. Vermis sen werde ich auch meine Kolleginnen und Kollegen des Generalsekretariates, viele weitere externe Ansprechpersonen und ganz besonders die FMH Kommunikationscrew. Was für die Medizin gilt, gilt genauso für die Kommunikation: Eine gute interdisziplinäre Zusammenarbeit sowie ein eingespieltes Team sind entscheidende Erfolgsfaktoren für eine gelingende Kommunikation. Nicht vermissen werde ich wohl den langen Arbeitsweg von meinem Wohnort Zürich nach Bern, auch wenn man sich über die Jahre daran gewöhnt und die Zeit zu nutzen weiss.

\section{Professionelle Ansprechpartner bei Kommunikationsanliegen}

Die Abteilung Kommunikation der FMH steht sowohl Mitgliedern als auch Medienschaffenden und Gesundheitsakteuren jederzeit für Fragen und Anliegen rund um ärztliche Themen zur Verfügung. Die stellvertretende Leiterin der Abteilung Kommunikation, Cornelia Steck, übernimmt bis zum Antritt der/-s Nachfolgerin/-s von Frau Wettstein die Ad-interimsLeitung. kommunikation[at]fmh.ch, 0313591153 